\title{
Disfluency Effects on Lexical Selection
}

\author{
Srdan Medimorec \\ Torin P. Young \\ Evan F. Risko \\ University of Waterloo
}

Corresponding author information:

Srdan Medimorec

Department of Psychology, University of Waterloo, 200 University Avenue West, Waterloo ON, Canada, N2L 3G1

smedimorec@uwaterloo.ca

+1 519-781-3691

Word count: 2,995

This work was supported by a Discovery Grant from the Natural Sciences and Engineering Research Council of Canada (NSERC) to EFR. 


\begin{abstract}
Recent research has suggested that introducing a disfluency in the context of written composition (i.e., typing with one hand) can increase lexical sophistication. In the current study, we provide a strong test between two accounts of this phenomenon, one that attributes it to the delay caused by the disfluency and one that attributes it to the disruption of typical finger-to-letter mappings caused by the disfluency. To test between these accounts, we slowed down participants' typewriting by introducing a small delay between keystrokes while individuals wrote essays. Critically, this manipulation did not disrupt typical finger-to-letter mappings. Consistent with the delay-based account, our results demonstrate that the essays written in this less fluent condition were more lexically diverse and used less frequent words. Implications for the temporal dynamics of lexical selection in complex cognitive tasks are discussed.
\end{abstract}

Keywords: language; disfluency; writing; lexical selection; lexical sophistication 
"That's not writing, that's typing." --Truman Capote

\section{Introduction}

Recent research has suggested that in some circumstances, introducing a disfluency can benefit performance (e.g., Ball, Klein, \& Brewer, 2014; Mueller \& Oppenheimer, 2014). A surprising example of this general phenomenon was recently reported in the context of written composition by Medimorec and Risko (2016), who found that decreasing transcription fluency (or typing speed) by having individuals type with one hand resulted in more lexically sophisticated essays. This finding is surprising theoretically because transcription fluency is typically thought to be positively correlated with writing quality as the automatization of transcription arguably affords the re-distribution of resources to higher level writing processes such as planning (Fayol, 1999; Kellogg, 1999). Thus understanding how transcription disfluency influences aspects of writing provides a unique opportunity to gain a deeper understanding of the basic mechanisms underlying lexical selection in written composition (and language use in general). Here we provide a strong test between two potential theoretical accounts of the effect of disfluency on lexical sophistication.

\subsection{Typing disfluency and cognition}

In Medimorec and Risko (2016), transcription disfluency was introduced by having participants typewrite essays using one hand (vs. standard typing). When essays were computationally analyzed, the results demonstrated that essays typewritten in the less fluent (or slower) condition were more lexically sophisticated (i.e., they exhibited increased lexically diversity and decreased word frequency). Medimorec and Risko (2016) interpreted these results as consistent with the idea that typewriting may be too fluent (Heidegger, 1992; Norman 2002). For example, Norman (2002) suggested that handwriting encouraged more thoughtful writing 
compared to typewriting because the former was slower. Medimorec and Risko (2016) proposed a time-based account of the effects of disfluency on lexical sophistication whereby decreasing transcription fluency allowed more time for lexical processes, such as lexical access, to unfold. Such a mechanism is consistent with the general underpinnings of most theories of lexical processing, which posit that accessing infrequent words takes more time than accessing more frequent words, both in speech and writing (Forster \& Davis, 1984; Navarrete, Basagni, Alario, \& Costa, 2006). For example, Crowe (1998) used a verbal fluency task to investigate lexical selection and found that participants first generated the more available frequent words followed by infrequent words. According to the time-based disfluency account, the critical variable in producing this effect on lexical sophistication should be the delay in transcription speed caused by disfluency.

While writing with one hand (relative to two) certainly slows down transcription, it also interferes with writing in other ways. For example, relatively skilled typewriting involves specific pairings of fingers and keyboard keys (Purcell, Napoliello, \& Eden, 2011), and switching to one-handed typing would disrupt those mappings. This disruption could in theory influence lexical selection during writing. For example, there is evidence that individuals exhibit different letter preferences when typing on a QWERTY keyboard across different input modalities depending on whether they use both hands or only one hand (Pelleg, Yom-Tov, \& Gabrilovich, 2015). Thus the way that we type can influence what we type. An effect on lexical sophistication would occur if lexical selection was influenced by the frequency of motor production (i.e., a bias towards selecting often typed words) and one-handed typing interfered with such an effect. In other words, the decreased lexical sophistication in the more fluent (or standard) typing condition could potentially be explained by a habitual combination of more 
rapid word access and the more automatized typing of those words (compared to infrequent words), with the latter being disrupted when we type one-handed.

In order to test between the two accounts described above, it is necessary to find a manipulation that could slow down regular (both-handed) typing without disrupting the fingerto-letter mappings. To this end, we had software developed that allowed us to introduce a delay between keypresses. Thus we could, relatively directly, control typing speed while individuals used their familiar two-handed typing (and thus maintained the same finger-to-letter relations across conditions). To our knowledge, this is the first time that the effects of such a manipulation have been reported.

In addition to the two accounts described above, we also examined the idea that fluency effects in composition could result from effects on participants' subjective task experience. Previous work has provided evidence that conscious experience of low effort or high speed, referred to as subjective fluency (Winkielman, Schwarz, Fazendeiro, \& Reber, 2003), can influence cognitive processing. For example, subjective fluency can elicit a positive affective reaction (Schwarz \& Clore, 1996) and facilitate creativity (Nadler, Rabi, \& Minda, 2010). Moreover, individuals can perceive the same objective experiences as more or less fluent depending on previous experience and expectations (Whittlesea, 1993). Thus many fluency effects can involve a subjective fluency component. To test this notion, participants' responses to the NASA Task Load Index (NASA-TLX; see Hart \& Staveland, 1988), a multidimensional scale providing measures of subjective fluency, were examined. Finally, since increased lexical sophistication represents one of the predictors of better essay quality (Crossley \& McNamara, 2011), we investigate whether disfluency effects on lexical sophistication extend to human essay quality judgements. 


\section{Method}

\subsection{Participants}

A total of 202 university students participated, but two participants did not complete the study. Sample size was determined using the effect size of .40 (based on Medimorec \& Risko, 2016), and power of .80 (Faul, Erdfelder, Buchner, \& Lang, 2009).

\subsection{Design}

A 2 (standard vs. keyboard delay condition) between-subject design was used.

\subsection{Stimuli and apparatus}

The essays were written using a QWERTY keyboard and software that controlled the minimum time delay between keystrokes. Based on piloting, we set this minimum delay to 100 ms. ${ }^{1}$ Subjective fluency measures were collected using NASA-TLX, which has scales that range from -10 (i.e., low) to +10 (i.e., high) for six dimensions: mental, physical, and temporal demand, performance, effort, and frustration. Keystroke activity was recorded using our delay application and the Inputlog key-logger (Leijten \& Van Waes, 2013).

\subsection{Procedure}

Participants wrote a timed (50 min) argumentative essay regarding cellphone use in schools (Medimorec \& Risko, 2016). Participants were asked to write at least 500 words and were informed that their essays would be graded. Participants were given a 3-minute practice to get familiarized with the keyboard by typing a sentence. After the writing task, participants filled out the NASA-TLX scale.

\subsection{Measuring descriptives and linguistic features of essays}

\footnotetext{
${ }^{1}$ Note that this does not necessarily lead to a $100 \mathrm{~ms}$ decrease in typing speed because the majority of keypresses in regular typing already exceed $100 \mathrm{~ms}$ ( 73\% in our standard condition). Thus the introduced delay could be roughly conceptualized as a removal of all sub $100 \mathrm{~ms}$ interword keypresses from standard typing.
} 
Transcription fluency was determined by calculating times between consecutive lower case letters recorded by our delay application. We removed $1.42 \%$ of keystrokes within individual participants exceeding 2.5 SD of the mean (for 199 participants; one participant used all caps).

Essays were analyzed using the Coh-Metrix text analyzer (McNamara, Graesser, McCarthy, \& Cai, 2014). We also report corresponding language indices using the Tool for the Automatic Analysis of Lexical Sophistication (TAALES; Kyle \& Crossley, 2015), and the Tool for the Automatic Analysis of Cohesion (TAACO; Crossley, Kyle, \& McNamara, 2015). We focus on two relatively independent lexical sophistication indices affected by the transcription fluency manipulation in Medimorec and Risko (2016), specifically, lexical diversity and word frequency.

\subsubsection{Lexical diversity}

Lexical diversity is the range of vocabulary in a text. Coh-Metrix computes the typetoken ratio (TTR; Templin, 1957), the measure of textual lexical diversity (MTLD; McCarthy \& Jarvis, 2010), and vocd-D (Malvern, Richards, Chipere, \& Duran, 2004). In general, these indices measure the ratio of unique words relative to the number of words in the text. Higher lexical diversity indicates greater lexical sophistication (McNamara et al., 2014). TTR results were also derived from TAACO.

\subsubsection{Word frequency}

Word frequency is a measure of how often individual words occur in the English language. Coh-Metrix derives the log-frequency for all words and the raw word frequency for content words from the CELEX database (Baayen, Piepenbrock, \& Gulikers, 1996). Word frequencies from the SUBTLEXus (Brysbaert \& New, 2009) and British National Corpus (BNC; 
BNC Consortium, 2007) databases are derived by TAALES. Texts with infrequent words are considered more lexically sophisticated (Crossley \& McNamara, 2011).

\subsection{Essay grading}

Three trained raters independently and blind to condition scored essays using a 6-point rating scale (based on the ACT Writing Test Scoring Rubric). Raters were trained to use the rubric by scoring two samples of argumentative essays from another corpus $(N=30)$ until the averaged interrater reliability reached $r>.50$. When the essays from the current study were scored, the interrater reliability was significant, $r(198)=.56, p<.001$. The mean score between the two raters with the highest correlation was used as the final score of each essay. If the differences between these two raters were $\geq 1$, then the score closest to the third rater's score was used.

\section{Results}

A series of one-way ANOVAs were performed with condition (standard vs. keyboard delay) as the factor and transcription fluency, lexical sophistication, subjective fluency, and essay quality as the dependent variables.

\subsection{Descriptive essay indices}

Condition affected transcription fluency such that it was more fluent in the standard condition, $F(1,197)=110.14, M S E=2796.13, p<.001, d=1.50$. While there were more words typed in the standard condition, $F(1,198)=6.35, M S E=14329.49, p=.013, d=.36$, essays in the delay condition contained longer words (letters and syllables per word), $F s>4.17, p s<.043$, $d s>.28$. There were no other differences (see Table 1).

Table 1. 
DISFLUENCY

Means, Standard Deviations, and Cohen's d (Cohen, 1988) of Essay Descriptive Measures Across the Two Conditions

\begin{tabular}{|c|c|c|c|c|c|}
\hline & \multicolumn{2}{|c|}{ Standard } & \multicolumn{2}{|c|}{ Delay } & \multirow{2}{*}{$\begin{array}{l}\text { Difference } \\
\text { Cohen's d }\end{array}$} \\
\hline Measure & $\mathrm{M}$ & $\mathrm{SD}$ & $\mathrm{M}$ & $\mathrm{SD}$ & \\
\hline Transcription Fluency & 223.37 & 58.47 & 302.05 & 46.55 & $1.50^{* * 3 * *}$ \\
\hline Number of Words & 593.66 & 138.56 & 551.01 & 97.27 & $.36^{* *}$ \\
\hline Word Length (letters) & 4.68 & .24 & 4.75 & .25 & $.29^{* *}$ \\
\hline Word Length (syllables) & 1.49 & .08 & 1.51 & .09 & $.31^{* *}$ \\
\hline Sentence Count & 25.91 & 6.48 & 25.39 & 6.55 & .08 \\
\hline Words per Sentence & 23.57 & 4.90 & 22.55 & 4.92 & .21 \\
\hline Paragraph Count & 4.12 & 1.71 & 4.15 & 1.72 & .02 \\
\hline
\end{tabular}

\subsection{Lexical sophistication}

Condition had a significant effect on type-token ratio, $F(1,198)=9.70, M S E=.002, p=$ $.002, d=.44$, and vocd-D (marginally), $F(1,198)=3.29, M S E=264.62, p=.071, d=.26$, such that they were higher in the delay condition. There was no effect on the measure of textual lexical diversity, $F(1,198)=2.13, M S E=239.96, p=.146, d=.21$, though the pattern of means was in the same direction. Moreover, condition had an effect on both word frequency indices such that they were lower in the delay condition, log word frequency-all words, $F(1,198)=4.49$, $M S E=.01, p=.035, d=.30$, raw word frequency-content words, $F(1,198)=4.74, M S E=.02, p$ $=.031, d=.31$ (see Table 2). The results were similar when lexical diversity and word frequency from TAACO and TAALES were used (see Table 3).

Table 2. 
Means, Standard Deviations, and Cohen's $d$ of Lexical Diversity and Word Frequency Across the Two Conditions, Coh-Metrix Indices

Standard Delay Difference

\begin{tabular}{|c|c|c|c|c|c|}
\hline Measure & $\mathrm{M}$ & SD & $\mathrm{M}$ & SD & Cohen's d \\
\hline Type-Token Ratio & .41 & .04 & .43 & .04 & $.44^{* *}$ \\
\hline Measure of Textual Lexical Diversity & 80.40 & 14.43 & 83.60 & 16.49 & .21 \\
\hline vocd-D & 87.06 & 14.81 & 91.23 & 17.61 & $.26^{*}$ \\
\hline Log Frequency All Words & 3.03 & .09 & 3.01 & .08 & $.30^{* *}$ \\
\hline Word Frequency Content Words (Raw) & 2.37 & .12 & 2.33 & .12 & $.31^{* *}$ \\
\hline
\end{tabular}

Table 3.

Means, Standard Deviations, and Cohen's d of Lexical Diversity and Word Frequency Across the Two Conditions, TAACO and TAALES Indices

\begin{tabular}{|c|c|c|c|c|c|}
\hline \multirow[b]{2}{*}{ Measure } & \multicolumn{2}{|c|}{ Standard } & \multicolumn{2}{|c|}{ Delay } & \multirow{2}{*}{$\begin{array}{l}\text { Difference } \\
\text { Cohen's d }\end{array}$} \\
\hline & $\mathrm{M}$ & $\mathrm{SD}$ & M & $\mathrm{SD}$ & \\
\hline Type-Token Ratio & .42 & .04 & .44 & .05 & $.46^{* * *}$ \\
\hline Log Frequency All Words (SUBTLEXus) & 4.44 & .14 & 4.40 & .13 & $.35^{* *}$ \\
\hline Log Frequency All Words (BNC) & 4.91 & .08 & 4.87 & .09 & $.37^{* *}$ \\
\hline Log Frequency Content Words (SUBTLEXus) & 3.77 & .17 & 3.71 & .18 & $.35^{* *}$ \\
\hline Log Frequency Content Words (BNC) & 4.26 & .10 & 4.22 & .12 & $.34^{* *}$ \\
\hline${ }^{*} p<.05$ level. & & & & & \\
\hline
\end{tabular}


Condition had an effect on two of the subjective fluency measures, namely physical demand, $F(1,198)=12.34, M S E=30.20, p=.001, d=.50$, and frustration, $F(1,198)=27.46$, $M S E=32.12, p<.001, d=.74$, such that they were higher in the delay condition. The other measures were unaffected, $F s<2.27, p s>.13, d s<.22$ (see Table 4).

Table 4.

Means, Standard Deviations, and Cohen's d of Subjective Fluency Measures Across the Two Conditions

\begin{tabular}{lccccc}
\hline & \multicolumn{2}{c}{ Standard } & \multicolumn{2}{c}{ Delay } & Difference \\
\hline Measure & M & SD & M & SD & Cohen's d \\
\hline Mental Demand & .16 & 4.80 & 1.10 & 4.71 & .20 \\
Physical Demand & -5.27 & 4.81 & -2.54 & 6.10 & $.50^{* *}$ \\
Temporal Demand & -1.82 & 5.05 & -1.74 & 5.43 & .02 \\
Performance & 2.26 & 4.51 & 1.31 & 4.96 & .20 \\
Effort & 1.12 & 4.13 & 1.98 & 3.96 & .21 \\
Frustration & -2.78 & 5.48 & 1.42 & 5.85 & $.74^{* * *}$ \\
\hline$* 0.05$ level. ${ }^{* * * *} p<.001$. & & & & &
\end{tabular}

\subsection{Essay quality}

There was no effect of condition on human-judged essay quality, $F(1,198)=.55, M S E$ $=.19, p=.458, d=.11$. Graders rated the essays written with the standard keyboard $(M=4.24$ $\mathrm{SD}=.42)$ as similar to those written with the delayed keyboard $(M=4.19 \mathrm{SD}=.45)$. Grades correlated positively with the number of words, $r(198)=.41$. Controlling for the number of words, lower word frequency was related to higher quality, raw word frequency-content words $B$ $=-.84, S E=.22, t(197)=-3.84, p<.001, r_{s}=-.24, \log$ word frequency-all words, $B=-1.16, S E$ 
$=.31, t(197)=-3.79, p<.001, r_{s}=-.24$, whereas lexical diversity measures were not related to quality judgements when controlling for the number of words, $B s<.58$, $t s<.81, p s<.44, r_{s} s<$ .06.

\subsection{Regression analysis}

We performed a regression analysis to determine the extent to which the effects of disfluency on lexical indices are related to differences in the number of words and subjective fluency (since condition affected the number of words, physical demand, and frustration). We entered condition $(0=$ standard, $1=$ delay $)$ and the number of words as the independent variables, and raw frequency-content words or type-token ratio (indices with the largest effect sizes) as the dependent variable. In the next step, we entered physical demand and frustration as additional independent variables. Effect sizes are semipartial correlations $\left(r_{s}\right)$.

Condition had a significant effect on raw frequency-content words, $B=-.040, S E=.02$, $t(197)=-2.27, p=.025, r_{s}=-.16$, and type-token ratio, $B=.012, S E=.01, t(197)=2.13, p=$ $.034, r_{s}=.13$, controlling for number of words. The effect remained significant with physical demand and frustration in the model for raw frequency-content words, $B=-.044, S E=.02$, $t(195)=-2.36, p=.019, r_{s}=-.17$, and type-token ratio (marginally), $B=.010, S E=.01, t(195)=$ $1.74, p=.083, r_{s}=.11$.

\section{Discussion}

The results of the current study provide clear evidence against the mapping disruption account of disfluency on lexical sophistication. In particular, by introducing a delay between consecutive key presses, we were able to slow typing without disrupting familiar finger-to-letter mappings. Despite the latter, we still observed a significant effect of disfluency on lexical sophistication. Specifically, the essays written in the keyboard delay condition were more 
lexically diverse and used less frequent words. This result is consistent with the time-based account suggested by Medimorec and Risko (2016) wherein slowing typing provides additional time for lower frequency alternatives and more alternatives to be activated in the lexical memory.

The transcription fluency manipulation also influenced the subjective estimates of physical demand and frustration. Interestingly, the effect of disfluency on lexical diversity (marginally) and word frequency remained after controlling for these effects. Thus, while introducing a disfluency in transcription clearly has a marked effect on composers' subjective experiences, there appears to be an effect of this disfluency on lexical sophistication that is independent of these effects. That said, it remains an interesting hypothesis to further pursue given that we only examined a limited number of subjective measures. In addition, we have focused on a specific set of lexical indices tied to lexical sophistication and there exists a large number of other lexical measures that could be influenced by introducing a transcription disfluency and the resulting subjective experiences (in the supplementary materials, we provide additional measures of lexical sophistication and measures from LIWC; Tausczik \& Pennebaker, 2010).

Lastly, the observed differences in lexical sophistication did not translate to an effect on subjective essay quality. There are a number of potential reasons for this result. First, it is possible that our measure of grading was not sufficiently sensitive. For example, the reliability between essay graders was not particularly high. Second, it is possible that while lexical sophistication is related to essay quality, the magnitude of the effect of transcription disfluency on lexical sophistication (here $d \sim .30$ ) is not large enough to translate into human-graded essay quality. Lastly, it is also possible that the previously reported relation between lexical 
sophistication and essay quality (e.g., Crossley \& McNamara, 2011) is confounded by some third variable. For example, rather than the use of lower frequency words causing higher essay scores, it might be that individuals who tend to use lower frequency words are superior on other aspects of writing that are more directly related to subjective essay quality. In the latter case, a manipulation that influences lexical sophistication but not these other aspects of writing would not be expected to translate into an effect on subjective essay quality.

The disfluency effects in written composition reported here diverge to some extent from previous research since we did not find a negative relation between transcription disfluency and writing quality reported previously. For example, when participants in Olive, Alves, and Castro (2009) wrote essays using a disfluent cursive uppercase calligraphy, they produced essays that were judged to be of lesser quality to those written in participants' own familiar calligraphy. Similarly, Alves, Castro, and Olive (2008) reported that slower typists produced texts that contained fewer different words compared to faster typists. Why the discrepancy? One hypothesis is that, unlike previous studies, the transcription disfluency manipulation in the current study did not introduce large working memory demands. Consistent with this notion, there was no significant effect of condition on perceived mental demand or performance. In addition, in an unpublished experiment using a dual-task technique, we found no difference between a standard typing condition and typing with a $100 \mathrm{~ms}$ delay with respect to participants' response time to a pseudorandom probe presented while they were writing. ${ }^{2}$ Not burdening working memory is important because lexical selection is likely tied to the availability of

\footnotetext{
${ }^{2}$ In a within-subject study, 33 participants were asked to respond to auditory probes while writing three timed $(7 \mathrm{~min}$ ) paragraph-length narratives in three different conditions (keyboard delay: 0,100 , or $300 \mathrm{~ms})$. In order to calculate baseline RTs, participants first executed only the RT task. There was a significant dual-task effect, $F(1.85,59.16)=125.89, M S E=.02, p<.001$, $\eta_{p}^{2}=.80$ but the effect was not larger in the $100 \mathrm{~ms}$ or $300 \mathrm{~ms}$ delay conditions compared to standard typing $(t s<1.65, p s>.10)$.
} 
working memory. Indeed, previous research has demonstrated that increasing working memory load decreases verbal fluency (Rosen \& Engle, 1997).

\section{Conclusion}

The current study provided a test between a time-based and a mapping disruption account of the effects of disfluency on written composition. The results were consistent with the former account. That is, by providing additional time for lexical processes to unfold, disfluency can increase lexical sophistication in written composition. In addition, we demonstrated effects of transcription disfluency on the subjective experience of that task (i.e., physical demand, frustration), but these effects did not appear to explain the effect of transcription fluency on lexical sophistication. Finally, the reported effects of disfluency on lexical sophistication did not translate to human-judged essay quality. 
Alves, R. A., Castro, S. L., \& Olive, T. (2008). Execution and pauses in writing narratives: Processing time, cognitive effort and typing skill. International Journal of Psychology, 43, 969-979.

Baayen, R. H., Piepenbrock, R., \& Gulikers, L. (1996). Celex2. Philadelphia, PA: Linguistic Data Consortium, University of Pennsylvania.

Ball, B. H., Klein, K. N., \& Brewer, G. A. (2014). Processing fluency mediates the influence of perceptual information on monitoring learning of educationally relevant materials. Journal of Experimental Psychology: Applied, 20,336-348.

British National Corpus Consortium. (2007). British national corpus version 3 (BNC XMLth ed.).

Brysbaert, M., \& New, B. (2009). Moving beyond Kučera and Francis: A critical evaluation of current word frequency norms and the introduction of a new and improved word frequency measure for American English. Behavior Research Methods, 41, 977-990.

Cohen, J. (1988). Statistical power analysis for the behavioral sciences $\left(2^{\text {nd }}\right.$ ed.) Hillsdale, NJ: Erlbaum.

Crossley, S. A., Kyle, K., \& McNamara, D. S. (2015). The tool for the automatic analysis of text cohesion (TAACO): Automatic assessment of local, global, and text cohesion. Behavior Research Methods, 1-11.

Crossley, S. A., \& McNamara, D. S. (2011). Understanding expert ratings of essay quality: CohMetrix analyses of first and second language writing. International Journal of Continuing Engineering Education and Life Long Learning, 21, 170-191. 
Crowe, S. F. (1998). Decrease in performance on the verbal fluency test as a function of time: Evaluation in a young healthy sample. Journal of Clinical and Experimental Neuropsychology, 20, 391-401.

Faul, F., Erdfelder, E., Buchner, A., \& Lang, A. G. (2009). Statistical power analyses using G* Power 3.1: Tests for correlation and regression analyses. Behavior Research Methods, 41, 1149-1160.

Fayol, M. (1999). From on-line management problems to strategies in written composition. In M. Torrance \& G. Jeffery (Eds.), The cognitive demands of writing: Processing capacity and working memory effects in text production (pp. 13-23). Amsterdam: Amsterdam University Press.

Forster, K. I., \& Davis, C. (1984). Repetition priming and frequency attenuation in lexical access. Journal of Experimental Psychology: Learning, Memory, and Cognition, 10, 680698.

Heidegger, M. (1992). Parmenides (A. Schuwer \& R. Rojcewicz, Trans.). Bloomington, IN: Indiana University Press.

Kellogg, R. T. (1999). Components of working memory in text production. In M. Torrance \& G. Jeffery (Eds.), The cognitive demands of writing: Processing capacity and working memory effects in text production (pp. 43-61). Amsterdam: Amsterdam University Press.

Kyle, K., \& Crossley, S. A. (2015). Automatically assessing lexical sophistication: Indices, tools, findings, and application. TESOL Quarterly, 49, 757-786.

Leijten, M., \& Van Waes, L. (2013). Keystroke logging in writing research using Inputlog to analyze and visualize writing processes. Written Communication, 30, 358-392. 
Malvern, D., Richards, B. J., Chipere, N., \& Duran, P. (2004). Lexical diversity and language development: Quantification and assessment. Basingstoke, UK: Palgrave Macmillan.

McCarthy, P. M., \& Jarvis, S. (2010). MTLD, vocd-D, and HD-D: a validation study of sophisticated approaches to lexical diversity assessment. Behavior Research Methods, 42, 381-392.

McNamara, D. S., Graesser, A. C., McCarthy, P. M., \& Cai, Z. (2014). Automated evaluation of text and discourse with Coh-Metrix. Cambridge, UK: Cambridge University Press.

Medimorec, S., \& Risko, E. F. (2016). Effects of disfluency in writing. British Journal of Psychology. doi: 10.1111/bjop.12177

Mueller, P. A., \& Oppenheimer, D. M. (2014). The Pen Is Mightier Than the Keyboard Advantages of Longhand Over Laptop Note Taking. Psychological Science, 25, 1-10.

Navarrete, E., Basagni, B., Alario, F. X., \& Costa, A. (2006). Does word frequency affect lexical selection in speech production? The Quarterly Journal of Experimental Psychology, 59, 1681-1690.

Norman, D. A. (2002). The design of everyday things. New York, NY: Basic books.

Olive, T., Alves, R. A., \& Castro, S. L. (2009). Cognitive processes in writing during pause and execution periods. European Journal of Cognitive Psychology, 21, 758-785.

Pelleg, D., Yom-Tov, E., \& Gabrilovich, E. (2015). On the Effect of Human-Computer Interfaces on Language Expression. arXiv preprint arXiv:1505.00092.

Purcell, J. J., Napoliello, E. M., \& Eden, G. F. (2011). A combined fMRI study of typed spelling and reading. Neuroimage, 55, 750-762.

Rosen, V. M., \& Engle, R. W. (1997). The role of working memory capacity in retrieval. Journal of Experimental Psychology: General, 126, 211-227. 
Schwarz, N., \& Clore, G. L. (1996). Feelings and phenomenal experiences. Social psychology: Handbook of basic principles, 2, 385-407.

Strömqvist, S. (1999). Production rate profiles. In S. Strömqvist, \& E. Ahlsén (Eds), The process of writing: A progress report (pp. 53-70). Gothenburg, Sweden: Department of Linguistics, University of Göteborg.

Tausczik, Y. R., \& Pennebaker, J. W. (2010). The psychological meaning of words: LIWC and computerized text analysis methods. Journal of Language and Social Psychology, 29, 2454.

Templin, M. (1957). Certain language skills in children: Their development and interrelationships. Minneapolis, MN: University of Minnesota Press.

Whittlesea, B. W. (1993). Illusions of familiarity. Journal of Experimental Psychology: Learning, Memory, and Cognition, 19, 1235-1253.

Winkielman, P., Schwarz, N., Fazendeiro, T, \& Reber, R. (2003). The hedonic marking of processing fluency: Implications for evaluative judgment. In J. Musch \& K.C. Klauer (eds.), The psychology of evaluation: Affective processes in cognition and emotion (pp. 189-217). Mahwah, NJ: Erlbaum. 


\section{Highlights}

- A time-based account of the effects of transcription disfluency on lexical sophistication is tested.

- Essays written with a slight keyboard delay were more lexically sophisticated.

- A transcription disfluency is argued to provide additional time for lexical processes to unfold. 